\title{
Surveillance nationale des entérovirus non poliomyélitiques au Canada :
} pourquoi est-ce important?

\author{
Booth $\mathrm{TF}^{1,2 *}$, Grudeski $\mathrm{E}^{1}$, McDermid $\mathrm{A}^{1,2}$ \\ ${ }^{1}$ Laboratoire national de microbiologie, Agence de la santé publique du Canada, Winnipeg (Manitoba) \\ ${ }^{2}$ Département de microbiologie médicale, Université du Manitoba, Winnipeg (Manitoba) \\ *Auteur-ressource : Tim.booth@phac-aspc.gc.ca
}

\section{Résumé}

Une éclosion à grande échelle de l'entérovirus D68 (EV-D68) a été détectée en association avec une maladie respiratoire chez l'enfant partout au Canada et aux États-Unis à l'automne 2014. La majorité des cas étaient d'intensité légère, mais certains ont été associés à des problèmes de santé plus graves nécessitant une hospitalisation, dont des cas s'accompagnant de symptômes neurologiques, y compris la paralysie, de même que trois décès en Colombie-Britannique. L'EV-D68 figure parmi de nombreux entérovirus, dont les virus Coxsackie, les échovirus et le poliovirus. À part le vaccin contre le poliovirus, aucun autre n'est offert pour prévenir les infections entérovirales, et aucun médicament antiviral n'a été approuvé pour les traiter. Plus de 46 sérotypes en circulation au Canada ont été identifiés au cours des 25 dernières années. Jusqu'en 2014, l'EVD68 était rare. Le génotypage effectué systématiquement par le Laboratoire national de microbiologie (LNM) du Canada a permis d'identifier seulement 85 isolats de l'EV-D68 entre 1991 et 2013, alors que 282 isolats ont été détectés entre juillet et octobre 2014. La complexité de l'épidémiologie de ces entérovirus démontre la nécessité d'en effectuer le génotypage, en vue de détecter les éclosions dans l'espace et dans le temps, de déterminer l'incidence relative et les effets de ceux-ci sur la population de même que pour enquêter sur les tendances évolutionnaires, notamment les événements de recombinaison, car on estime que celles-ci jouent un rôle important dans la variation des souches et l'apparition de souches épidémiques. En particulier, il est important d'effectuer des tests virologiques lors de cas inhabituels de paralysie chez l'enfant et de procéder au génotypage ainsi qu'au séquençage de tout virus identifié. On encourage la soumission d'échantillons (cultures virales, selles, liquide céphalorachidien ou échantillons des voies respiratoires) de ce type de cas au LNM.

\section{Introduction}

L'entérovirus D68 (EV-D68) a retenu l'attention du public aux mois d'août et d'octobre 2014 lorsqu'une éclosion à grande échelle a été détectée en association avec une maladie respiratoire chez l'enfant partout au Canada et aux États-Unis (1-6). D'intensité légère, la majorité des cas comprenaient des symptômes pseudogrippaux, tels que la fièvre, la toux, la rhinite, la pharyngite, la bronchite et la myalgie. Une partie de ces cas d'infection à EVD68 ont été associées à une maladie plus grave nécessitant une hospitalisation. Parmi les cas d'hospitalisation, on compte la pneumonie, la bronchiolite et les difficultés respiratoires; l'asthme sous-jacent semblait par ailleurs être le principal facteur de risque. Certains cas ont présenté des symptômes neurologiques, y compris la paralysie, et trois décès ont été signalés en Colombie-Britannique (6). Aux États-Unis, les Centers for Disease Control and Prevention (CDC), qui ont analysé environ 2600 échantillons d'EV-D68 à la fin de l'été 2014, ont trouvé qu'environ $36 \%$ d'entre eux présentaient le virus; parmi les cas d'infection, 12 patients sont décédés et la présence du virus a été confirmée chez 1152 patients vivant dans 49 États (7). Au Canada, le Laboratoire national de microbiologie (LNM) a analysé 970 échantillons d'EV-D68 et identifié 282 cas positifs du mois d'août au mois d'octobre 2014. Cela donne à penser que l'éclosion d'EV-D68 aux États-Unis pourrait être liée à l'augmentation du nombre de cas de myélite flasque aiguë, une maladie neurologique inexpliquée qui entraîne la faiblesse des membres chez l'enfant et qui a coïncidé avec la détection accrue de l'EV-D68 (8). L'objectif de cet article consiste à fournir un aperçu de la complexité de l'étiologie et de l'épidémiologie des maladies entérovirales autres que la poliomyélite, ainsi qu'à déterminer les raisons pour lesquelles il est important de surveiller ce groupe d'infections. II faudra effectuer une surveillance épidémiologique fondée sur des analyses de laboratoire, en vue de vérifier les liens possibles entre l'infection entérovirale et les maladies paralytiques qui apparaissent. 
Les entérovirus appartiennent à la famille des picornavirus, un vaste groupe de divers virus à petit acide ribonucléique (ARN) caractérisés par un seul brin d'ARN génomique positif; cette famille comprend quatre espèces principales - A, B, C et $D$. Les souches du poliovirus font partie du groupe $C$ (9). Le poliovirus partage donc de nombreuses caractéristiques biochimiques et physiques avec d'autres entérovirus humains (p. ex. les virus Coxsackie, les échovirus et les rhinovirus, ces derniers étant ceux qui causent le rhume). Les entérovirus non poliomyélitiques causent de nombreux syndromes cliniques, en particulier la maladie infectieuse piedsmains-bouche, la méningite à liquide clair, la paralysie flasque, la myocardite, la pneumonie et la maladie respiratoire, la fièvre, la gastro-entérite, l'hépatite et la pancréatite. La plupart de ces infections touchent les enfants de manière disproportionnée, et nombre d'entre elles sont mortelles. II est aussi probable que les infections entérovirales soient hautement sous-diagnostiquées, car les tests de laboratoire ne sont pas souvent réalisés. À part le vaccin contre le poliovirus, aucun autre n'est offert pour prévenir les infections entérovirales, et aucun médicament antiviral n'a été approuvé pour les traiter. Au Canada, comme dans de nombreuses autres régions tempérées du monde, la période de pointe des infections entérovirales a lieu vers la fin de l'été ainsi qu'au cours des premiers mois de l'automne (Figure 1). Les entérovirus sont habituellement transmis par voie fécale/orale, par l'intermédiaire d'eau contaminée et lors de contacts étroits avec les personnes infectées. Les entérovirus peuvent être détectés dans des échantillons de selles, des échantillons provenant des voies respiratoires ainsi que dans des échantillons de liquide céphalorachidien de personnes infectées (10). De nombreux entérovirus, y compris l'EV-D68, sont également évacués dans les sécrétions respiratoires, ce qui tend à indiquer que les voies respiratoires seraient aussi ciblées par la transmission de l'EV-D68. Jusqu'à présent, l'on n'a jamais signalé la présence d'EV-D68 dans le système nerveux, qui pourrait fournir un mécanisme hypothétique pour expliquer la possibilité des effets neuropathogènes, y compris la paralysie.

Figure 1 : Variations saisonnières des infections échovirales au Canada, de 1990 à 2009ª

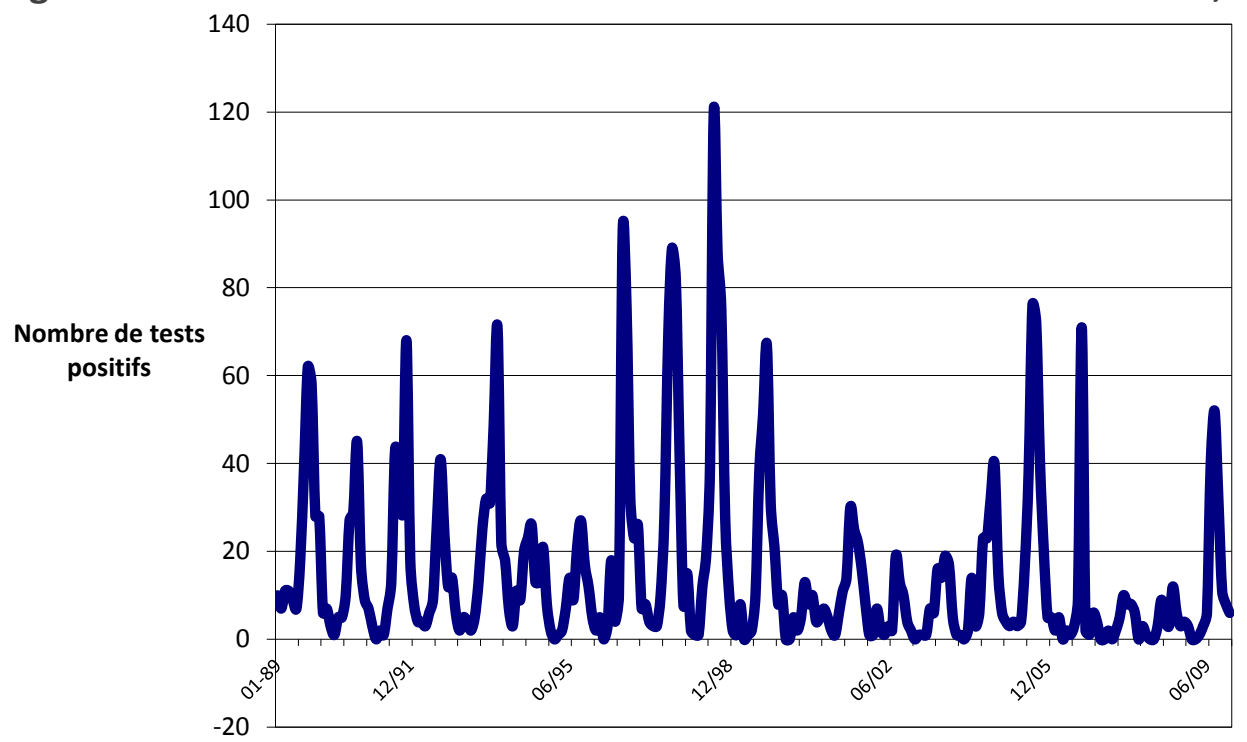

${ }^{a}$ Données mensuelles de tests positifs d'après le Programme canadien de déclaration des maladies à virus (11).

\section{Surveillance des entérovirus au Canada et l'apparition de l'EV-D68}

L'EV-D68 a rarement été identifié au Canada entre 1990 et 2014. Le génotypage systématiquement effectué par le National Centre for Enteroviruses au Laboratoire national de microbiologie de l'Agence de la santé publique du Canada a permis d'identifier seulement 85 isolats d'EV-D68, alors que 282 cas positifs sur 970 échantillons testés ont été observés entre juillet et octobre 2014 (Figure 2). L'EV-D68 est habituellement associé à des infections respiratoires et à des maladies s'apparentant à un rhume d'intensité légère, mais selon certains, on le signale parfois en association avec des maladies respiratoires plus aiguës chez l'enfant et, rarement, en association avec une maladie du système nerveux central (12). Des éclosions d'infections à EV-D68 chez l'enfant ont été détectées en 2009 et en 2010 en de nombreux endroits du monde entier (12-19). Au Canada, certaines données 
issues du génotypage effectué en laboratoire indiquent que des éclosions mineures d'EV-D68 sont aussi survenues durant les années 2009 (15 cas) et 2010 (23 cas) (Figure 2).

Figure 2 : Nombre de cas d'infections à EV-68 identifiés au Canada par génotypage moléculaire au Laboratoire national de microbiologie, de 2004 à 2014

Nombre de cas déterminés du génotype EV-D68

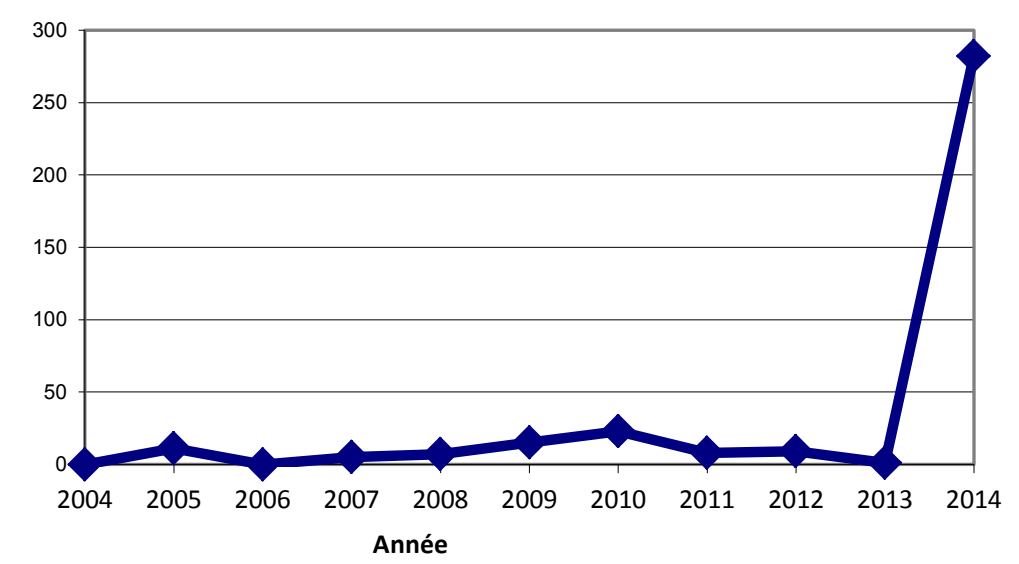

Le LNM effectue systématiquement le génotypage des échantillons d'entérovirus dans le cadre de ses services diagnostiques; ces tests présentent aussi l'avantage de fournir des données de surveillance sur l'incidence de différents sérotypes entéroviraux au Canada au fil des ans, en plus de permettre d'identifier rapidement toute éclosion inhabituelle. Plus de 46 sérotypes fréquemment en circulation au Canada ont été identifiés au cours des 25 dernières années. Les données sur les 20 entérovirus les plus fréquents apparaissent dans le Tableau 1. Une analyse annuelle comparative montre les sommets et les creux périodiques de chacun des sérotypes :

l'échovirus 30 est l'entérovirus non poliomyélitique le plus couramment en circulation au Canada. On a d'ailleurs connu des éclosions majeures de celui-ci en 1998, en 2006 et en 2009. II y a eu une éclosion des l'échovirus 7 en 2002 et la présence de l'échovirus 5 était à son sommet en 2007. Le virus Coxsackie A16 (CV-A16) représente la cause la plus courante de la maladie infectieuse pieds-mains-bouche et le CV-A9, qui est aussi couramment en circulation au Canada, est associé à ces éclosions de maladie. C'est d'ailleurs au cours de l'année 2003 qu'on l'a identifié le plus souvent en laboratoire. 
Tableau 1 : Nombre d'isolements de virus pour les 20 génotypes entéroviraux les plus prévalents au Canada de 1991 à 2013 relevé dans le cadre de la surveillance menée par le National Centre for Enteroviruses, Laboratoire national de microbiologie

\begin{tabular}{|l|r|r|}
\hline \multicolumn{1}{|c|}{ Sérotype entéroviral } & $\begin{array}{c}\text { Nombre total } \\
\text { d'isolements }\end{array}$ & \% du total \\
\hline Échovirus 30 (E-30) & 408 & 15,5 \\
\hline Virus Coxsackie A16 (CV-A16) & 196 & 7,5 \\
\hline Échovirus 11 (E-11) & 183 & 6,9 \\
\hline Virus Coxsackie (CV-A9) & 177 & 6,7 \\
\hline Échovirus 18 (E-18) & 149 & $5,6 \%$ \\
\hline Échovirus 25 (E-25) & 137 & 5,2 \\
\hline Échovirus 9 (E-9) & 116 & 4,4 \\
\hline Virus Coxsackie (CV-B4) & 111 & 4,2 \\
\hline Virus Coxsackie (CV-B3) & 104 & 3,9 \\
\hline Entérovirus A71 (EV-A71) & 101 & 3,8 \\
\hline Virus Coxsackie (CV-B2) & 98 & 3,7 \\
\hline Virus Coxsackie (CV-B5) & 97 & 3,6 \\
\hline Échovirus 6 (E-6) & 97 & 3,6 \\
\hline Entérovirus D68 (EV-D68) & 85 & 3,2 \\
\hline Échovirus 7 (E-7) & 83 & 3,1 \\
\hline Virus Coxsackie (CV-B1) & 80 & 3,0 \\
\hline Échovirus 5 (E-5) & 77 & 2,9 \\
\hline Échovirus 4 (E-4) & 58 & 2,2 \\
\hline Échovirus 22 & 45 & 1,7 \\
\hline Échovirus 3 (E-3) & 34 & \\
\hline
\end{tabular}

\section{À la défense de la surveillance}

Le génotypage est nécessaire en raison de la complexité de l'épidémiologie de ces entérovirus liée tant à la grande variété des génotypes présents dans la population qu'aux diverses maladies qui y sont associées. L'objectif consiste à détecter les éclosions dans l'espace et dans le temps, afin d'en déterminer l'incidence relative et les effets sur la population. De plus, cette surveillance renseigne sur les infections nouvelles, telles que celles à EV-A71 et à EV-D68, en plus d'offrir la possibilité de suivre l'évolution génétique des souches pouvant être associées à une augmentation de la pathogénicité. En particulier, il sera important d'effectuer des tests virologiques lors de cas inhabituels de paralysie chez l'enfant et de procéder au génotypage ainsi qu'au séquençage de tout virus identifié. Nous encourageons la soumission d'échantillons obtenus dans de tels cas à l'équipe responsable des entérovirus au LNM. Idéalement, ces échantillons seraient des cultures virales, mais le liquide céphalorachidien, les selles et les échantillons provenant des voies respiratoires conviennent également. Nous devons également demeurer vigilants à l'égard du poliovirus, maintenant que nous nous trouvons à un moment décisif en ce qui a trait à l'éradication de la poliomyélite à l'échelle mondiale. 


\section{Paralysie flasque aiguë et entérovirus non poliomyélitique}

La paralysie flasque aiguë correspond à l'apparition soudaine d'une réduction du tonus musculaire et d'une faiblesse musculaire sans cause apparente (telle qu'un traumatisme). Elle se caractérise habituellement par des membres relâchés et souples ainsi que par un manque de force nécessaire au mouvement et au contrôle des muscles. Celle-ci pourrait être causée par le syndrome de Guillain-Barré (un trouble auto-immun touchant le système nerveux périphérique) ainsi que par un certain nombre d'agents, y compris le poliovirus, des entérovirus non poliomyélétiques, des échovirus, l'adénovirus, le virus du Nil occidental ou encore par une infection à Campylobacter. Tout le corps peut être touché - et non seulement les membres -, ce qui pourrait entraîner de difficultés à respirer, la suffocation et la mort. Ainsi, un syndrome clinique très vaste, la paralysie flasque aiguë peut avoir un éventail de différentes causes et s'apparenter à d'autres syndromes neurologiques, notamment la myélite transverse ou antérieure et la névrite traumatique (20).

C'est un fait bien connu que les entérovirus non poliomyélitiques, tels que les virus Coxsackie, les échovirus et nombre d'autres entérovirus, dont les types $70,71,89,90,91,96,99,102$ et 114 , sont associés à de nombreuses manifestations neurologiques cliniques, telles que l'encéphalite, la méningite et la maladie paralytique, y compris des syndromes s'apparentant à la paralysie flasque aigu (21). Parmi ces virus, on a remarqué que l'EV-A71 était le plus couramment associé à la paralysie flasque d'origine autre que le poliovirus (20) ainsi qu'à des maladies neurologiques, notamment l'encéphalomyélite mortelle (22). L'EV-A71 est apparu durant les années 1990 comme un agent causant d'importantes éclosions périodiques de la maladie infectieuse pieds-mains-bouche chez l'enfant en Chine et dans d'autres parties d'Asie (23), et les sous-génotypes viraux en cause dans ces éclosions se sont également propagés en Asie du Sud-Est et en Australie. Une éclosion de l'EV-A71 à Taiwan en 1998 a causé 1,5 million d'infections et entraîné 78 décès (23). Une éclosion particulièrement importante de l'EV-A71 est survenue en Chine en 2009; même si le nombre total de cas n'a pas été signalé dans l'ensemble des provinces, il est apparu clair qu'une petite proportion de ceux-ci ont présenté des complications graves, notamment des symptômes neurologiques, et ont entraîné la mort (24). Néanmoins, les cas graves d'infection à l'EV-A71, en particulier ceux qui ont des atteintes neurologiques, sont relativement rares, et la plupart des enfants touchés se rétablissent entièrement. Les antiviraux efficaces contre cette classe de virus sont encore au stade de développement. Des efforts sont déployés pour mettre au point des vaccins afin de lutter contre les éclosions d'EV-A71 toujours en cours en Asie. En plus des éclosions de la maladie infectieuse piedsmains-bouche, les infections causées par des entérovirus autres non poliomyélitiques causent probablement aussi environ $50 \%$ de tous les cas de méningite à liquide clair (25). Par conséquent, nous disposons déjà d'un grand nombre de données selon lesquelles les infections entérovirales peuvent provoquer des maladies neurologiques, y compris la paralysie.

Une étude pilote menée au Canada durant le mois de septembre 2014 dans sept provinces et territoires a permis de recueillir des données épidémiologiques provenant de 268 cas d'hospitalisation causés par une infection à EVD68, parmi lesquels seulement 3 cas neurologiques ont été observés et aucun décès n'a été constaté (26). La rareté de ces cas neurologiques signifie que des études populationnelles beaucoup plus vastes devront être menées pour étudier davantage le lien possible entre l'EV-D68 et la paralysie. Mis à part la poliomyélite, les autres infections entérovirales constituent une difficulté, car leur déclaration n'est pas obligatoire au Canada, de sorte que le véritable nombre de cas confirmés en laboratoire ne peut être obtenu. De plus, la véritable incidence des infections est probablement beaucoup plus élevée que le nombre de cas confirmés en laboratoire, car la majorité des infections ne sont jamais soumis à des tests virologiques. Ainsi, il sera important de poursuivre cette surveillance améliorée au cours de la prochaine saison des entérovirus afin de déterminer le retour de l'EV-D68 en 2015 et, dans l'affirmative, de mesurer son effet sur la population, en particulier les enfants. Des cas plus graves d'infection entérovirale non poliomyélitique pourraient également être repérés par l'intermédiaire du système canadien de surveillance de la paralysie flasque aiguë ou du programme national de surveillance des génotypes entéroviraux du LNM. II est donc important de continuer à utiliser ces systèmes de surveillance virologique fondés sur des analyses de laboratoire afin de pouvoir détecter de nouvelles maladies entérovirales éventuelles au Canada. 


\section{Conclusion}

L'identification des associations ou des liens de cause à effet entre les éclosions de maladies entérovirales et d'autres maladies plus graves, telles que la paralysie, présente des difficultés que seule une surveillance additionnelle à grande échelle permettrait de surmonter. Cela doit comprendre la collecte d'un grand ensemble de données cliniques et épidémiologiques, de même que des tests de laboratoire, dont le typage et le séquençage moléculaires des virus. Bien qu'il n'existe aucun lien établi entre l'EV-D68 et la paralysie chez l'enfant, il est bien connu que les entérovirus sont des agents associés aux maladies neurologiques et à la paralysie dans une petite proportion de cas d'infections. L'apparition d'un entérovirus, l'EV-D68, qui était autrefois rarement identifié en tant que cause de maladie, soit à titre d'agent pouvant causer des éclosions à grande échelle de maladies respiratoires d'intensité légère à grave, constitue un nouveau risque pour la santé, qui nécessite des études continues et une surveillance étroite. L'augmentation du nombre d'échantillons soumis au typage entéroviral et l'amélioration du niveau des données épidémiologiques cliniques contenues dans ces soumissions constituent une des prochaines étapes à suivre. Les échantillons idéaux pour effectuer les tests d'entérovirus sont les cultures virales, les selles, le liquide céphalorachidien, ou encore les écouvillons ou les aspirats provenant des voies respiratoires. La surveillance des entérovirus non poliomyélitiques contribue à améliorer la confiance du statut du Canada en tant que pays sans poliomyélite, à déterminer l'incidence et les effets des entérovirus sur la santé ainsi qu'à surveiller l'apparition de nouveaux agents pouvant poser un nouveau risque pour la santé, tels que les virus EV-D68 et EV-A71.

\section{Remerciements}

Les auteurs remercient Rhiannon Huzarewich, Lily MacDonald, Michelle Gusdal et Russel Mandes pour leur extraordinaire assistance technique. Nous soulignons également le soutien offert par les laboratoires de santé publique et des hôpitaux canadiens.

\section{Financement}

Le programme sur les entérovirus a été financé par l'Agence de la santé publique du Canada.

\section{Conflit d'intérêts}

Aucun.

\section{Références}

(1) Midgley CM, Jackson MA, Selvarangan R, Turabelidze G, Obringer E, Johnson D, et al. Severe respiratory illness associated with enterovirus D68-Missouri and Illinois, 2014. MMWR. 2014;63(36):798-9.

(2) Hasegawa S, Hirano R, Okamoto-Nakagawa R, Ichiyama T, Shirabe K. Enterovirus 68 infection in children with asthma attacks: Virus-induced asthma in Japanese children. Allergy. 2011;66(12):1618-20. doi: 10.1111/j.13989995.2011.02725.x.

(3) Human enterovirus D68-North America (20): Update. ProMED. 2014 Dec 15. Archive No. 20141215.3035008. http://www.promedmail.org/direct.php?id=3035008

(4) Pastula DM, Aliabadi N, Haynes AK, et al. Acute neurologic illness of unknown etiology in children-Colorado, August-September 2014. MMWR. 2014;63(40):901-2. doi: mm6340a5 [pii].

(5) Fonseca K, Kellner JD, Talbot J, et al. Human enterovirus 68-Canada: (Alberta). ProMED. 2014 Sep 16 [cited 2015 Jan 6]. Archive No. 20140916.2775534. http://www.promedmail.org/direct.php?id=2775534

(6) BC Centre for Disease Control. British Columbia Influenza Surveillance Bulletin. Influenza Season 2014-15, Number 11, Weeks 51-52, December 14 to 27, 2014 [cited 2015 Jan 20]. http://www.bccdc.ca/NR/rdonlyres/B8298409-17F2-48B3-B146519D40AB5A06/0/InfluBulletin_Number11_Weeks5152_201415.pdf

(7) Centers for Disease Control and Prevention (CDC). Enterovirus D68 in the United States, 2014 [updated 2014 Oct 14; cited 2015 Jan 6]. http://www.cdc.gov/non-polio-enterovirus/outbreaks/EV-D68-outbreaks.html

(8) Roos R, Center for Infectious Disease Research and Policy (CIDRAP). CDC: Link between polio-like illness and EVD68 looks likely. CIDRAP News. 2014 Dec 2.

http://www.cidrap.umn.edu/news-perspective/2014/12/cdc-link-between-polio-illness-and-ev-d68-looks-likely 
(9) van Regenmortel MHV, et al., editors. International Union of Microbiological Societies, Virology Division. Virus taxonomy: Seventh report of the International Committee on Taxonomy of Viruses. San Diego: Academic Press; 2001.

(10) Jaramillo-Gutierrez G, Benschop KS, Claas EC, de Jong AS, van Loon AM, Pas SD, Pontesilli O, Rossen JW, Swanink CM, Thijsen S, van der Zanden AG, van der Avoort HG, Koopmans MP, Meijer A. September through October 2010 multi-centre study in the Netherlands examining laboratory ability to detect enterovirus 68, an emerging respiratory pathogen. J Virol Methods. 2013 Jun;190(1-2):53-62. doi: 10.1016/j.jviromet.2013.02.010. Epub 2013 Mar 1.

(11) Laboratory evidence of human viral and selected non-viral infections in Canada 1989 to 1996. CCDR. 1998 Oct;24S7. http://www.collectionscanada.gc.ca/webarchives/20071207052454/http://www.phacaspc.gc.ca/publicat/ccdr-rmtc/98pdf/24s7e.pdf. Disponible en français:http://www.collectionscanada.gc.ca/webarchives/20071213051449/http://www.phac-aspc.gc.ca/publicat/ccdrrmtc/98pdf/24s7f.pdf

(12) Kaida A, Kubo H, Sekiguchi J, et al. Enterovirus 68 in children with acute respiratory tract infections, Osaka, Japan. Emerg Infect Dis. 2011;17(8):1494-7. doi: 10.3201/eid1708.110028.

(13) Ikeda T, Mizuta K, Abiko C, et al. Acute respiratory infections due to enterovirus 68 in Yamagata, Japan between 2005 and 2010. Microbiol Immunol. 2012;56(2):139-43. doi: 10.1111/j.1348-0421.2012.00411.x.

(14) Meijer A, Benschop KS, Donker GA, van der Avoort HG. Continued seasonal circulation of enterovirus D68 in the Netherlands, 2011-2014. Eurosurveillance. 2014 Oct 23;19(42). http://www.eurosurveillance.org/ViewArticle.aspx?Articleld=20935

(15) Meijer A, van der Sanden S, Snijders BE, et al. Emergence and epidemic occurrence of enterovirus 68 respiratory infections in the Netherlands in 2010. Virology. 2012;423(1):49-57. doi: 10.1016/j.virol.2011.11.021.

(16) Jacobson LM, Redd JT, Schneider E, et al. Outbreak of lower respiratory tract illness associated with human enterovirus 68 among American Indian children. Pediatr Infect Dis J. 2012;31(3):309-12. doi:

10.1097/INF.0b013e3182443eaf.

(17) Centers for Disease Control and Prevention (CDC). Clusters of acute respiratory illness associated with human enterovirus 68-Asia, Europe, and United States, 2008-2010. MMWR. 2011;60(38):1301-4. doi: mm6038a1.

(18) Imamura T, Fuji N, Suzuki A, et al. Enterovirus 68 among children with severe acute respiratory infection, the Philippines. Emerg Infect Dis. 2011;17(8):1430-5. doi: 10.3201/eid1708.101328.

(19) Ayscue P, Van Haren K, Sheriff H, Waubant E, Waldron P, Yagi S, Yen C, Clayton A, Padilla T, Pan C, Reichel J, Harriman K, Watt J, Sejvar J, Nix WA, Feikin D, Glaser C. Acute flaccid paralysis with anterior myelitis-California, June 2012-June 2014. MMWR. 2014 Oct 10;63(40):903-6.

(20) Rao CD, Yergolkar P, Shankarappa KS. Antigenic diversity of enteroviruses associated with nonpolio acute flaccid paralysis, India, 2007-2009. Emerg Infect Dis. 2012 Nov; 18(11). doi: 10.3201/eid1811.111457. http://dx.doi.org/10.3201/eid1811.111457

(21) Rhoades RE, Tabor-Godwin JM, Tsueng G, Feuer R. Enterovirus infections of the central nervous system. Virology. 2011 Mar 15;411(2):288-305. doi: 10.1016/j.virol.2010.12.014. Epub 2011 Jan 20.

(22) Lum LC, Wong KT, Lam SK, Chua KB, Goh AY, Lim WL, Ong BB, Paul G, AbuBakar S, Lambert M. Fatal enterovirus 71 encephalomyelitis. J Pediatr. 1998 Dec;133(6):795-8.

(23) Wu W-H, Kuo T-C, Lin Y-T, Huang S-W, Liu H-F, et al. Molecular epidemiology of enterovirus 71 infection in the central region of Taiwan from 2002 to 2012. PLoS One. 2013 Dec 13;8(12):e83711. doi:10.1371/journal.pone.0083711. http://journals.plos.org/plosone/article?id=10.1371/journal.pone.0083711

(24) BBC News. China on alert as virus spreads. 2008 May 3 [cited 2015 Jan 6]. http://news.bbc.co.uk/2/hi/asia-pacific/7381741.stm

(25) Michos AG, Syriopoulou VP, Hadjichristodoulou C, Daikos GL, Lagona E, Douridas P, Mostrou G, Theodoridou M. Aseptic meningitis in children: Analysis of 506 cases. PLoS One. 2007 Aug 1;2(7):e674

(26) Edwin JJ, Reyes Domingo F, Booth TF, et al. Surveillance summary of hospitalized paediatric enterovirus D68 cases in Canada, September 2014. CCDR. 2015 Feb 20;41S1:2-8. Disponible en français: Résumé de la surveillance des cas pédiatriques d'entérovirus D68 hospitalisés au Canada, septembre 2014: RMTC. 2015 fév 20;41S1 :2-9. 